Ann. Biol. anim. Bioch. Biophys., I964, 4 (I), 9I-93.

\title{
ACTION DE LA PILOCARPINE SUR LA COMPOSITION DU SPERME DE TAUREAU
}

\author{
J. P. SIGNORET \\ avec la collaboration technique de Colette Lavenet et P. Evrard \\ Laboratoire de Physiologie de la Reproduction, \\ Centre national de Recherches zootechniques, Jouy-en-Josas (Seine-et-Oise)
}

SOMMAIRE

Dix taureaux ont reçu $3^{\circ}$ minutes avant la collecte une injection sous-cutanée, soit de $200 \mathrm{mg}$ de nitrate de pilocarpine en solution à $\mathrm{r}$ p. Ioo dans l'eau distillée, soit d'un volume équivalent de sérum physiologique stérile.

Aucun changement n'a été observé dans le comportement sexuel tandis que la composition de l'éjaculat subissait des modifications importantes : le volume passe de 2,8 à $5 \mathrm{ml}$, le nombre total de spermatozoïdes de 2,5 à 3,8 milliards.

L'innervation parasympathique du tractus génital semble donc jouer un rôle essentiel dans les mécanismes de l'éjaculation.

\section{IN'TRODUC'TION}

L'atropine réduit notablement le volume de sperme obtenu lorsqu'elle est injectée avant la collecte aussi bien chez le Verrat (DzIUK, I959) que chez le Taureau (Signoreit, I962). Cet effet semble dî surtout à une action inhibitrice sur les sécrétions accessoires puisque le nombre total de spermatozoïdes obtenus est inchangé (Verrat) ou faiblement réduit (Taureau). Il est intéressant d'observer les résultats obtenus avec des substances ayant un effet opposé à l'action parasympaticolytique del'atropine. DzIuk et NorTon (I962) ont essayé sans succès chez le Lapin le carbachol et la dibenamine, tandis que BAKER, Van Demark et Graves (I963) ont obtenu avec la pilocarpine une augmentation du volume de l'éjaculat et du nombre des spermatozoïdes chez le Taureau. Nous avions nous-mêmes entrepris une expérimentation comparable lorsque ces résultats ont été publiés. 


\section{MATÉRIEI, E'T TECHNIQUES}

Dix taureaux ont été utilisés, tous étaient âgés de trois ans au début de l'expérience et issus de la première génération d'un croisement mâle Frison $\times$ femelle Normande. Les collectes de sperme ont eu lieu deux fois par semaine à raison d'un seul éjaculat par Taureau. Le protocole expérimental et les techniques utilisées ont été analogues à ceux décrits lors de notre expérimentation sur l'atropine (SIGNORET, I962) :

Une dose de $200 \mathrm{mg}$ de nitrate de pilocarpine diluée dans $20 \mathrm{ml}$ d'eau distillée était injectée par voie sous-cutanée 3 o minutes avant le début de la période d'excitation précédant la collecte. La solution était préparée immédiatement avant chaque série d'injections.

Les témoins recevaient le même volume de sérum physiologique stérile.

La collecte du sperme était faite au vagin artificiel après un délai d'excitation de 5 minutes et les contrôles suivants effectués :

- motilité des spermatozoïdes notée subjectivement de o à 4 ;

- volume mesuré au $\mathrm{i} / \mathrm{ro}$ de $\mathrm{ml}$;

- concentration en spermatozoïdes déterminée par néphélométrie ;

- taux de spermatozoïdes morts déterminé par coloration différentielle à l'éosine-nigrosine (BLOM, I950).

Les résultats des collectes effectuées à la deuxième monte et de celles où du sperme avait été perdu n'ont pas été retenus.

Les animaux ont été répartis en deux groupes :

$\left.\mathrm{I}^{0}\right)$ un groupe expérimental recevant alternativement une injection de pilocarpine et une injection de sérum physiologique, afin d'éviter un effet cumulatif de la drogue ;

$2^{\circ}$ ) un groupe témoin recevant une injection de sérum physiologique avant chaque collecte. Une permutation circulaire des traitements a eu lieu toutes les quatre semaines de sorte que chaque animal reçut pendant huit semaines le traitement expérimental et quatre semaines le traitement témoin.

\section{RÉSULTATS E'T DISCUSSION}

Nous n'avons observé aucune différence dans les réactions des animaux témoins et traités, si ce n'est une abondante salivation chez ces derniers; le comportement sexuel n'a pas été perturbé : tous ont chevauché le boute-en-train dans les trente secondes dès que la possibilité leur en était laissée, et ont éjaculé normalement.

Les caractéristiques du sperme obtenu sont présentées dans le tableau ci-dessuus.

\begin{tabular}{|c|c|c|c|c|c|c|c|}
\hline & $\begin{array}{c}\text { Dose de } \\
\text { Pilocarpine } \\
\text { mg }\end{array}$ & $\begin{array}{c}\text { Nombre de } \\
\text { collectes }\end{array}$ & $\begin{array}{l}\text { Volume } \\
\text { moyen } \\
(\mathrm{ml})\end{array}$ & $\begin{array}{c}\text { Motilité } \\
\text { (notée sur 4) }\end{array}$ & $\begin{array}{l}\text { Concentration } \\
\left(\times \quad 1000 / \mathrm{mm}^{3}\right)\end{array}$ & $\begin{array}{l}\text { Nombre total } \\
\left(\begin{array}{l}\left.\times 10^{6}\right)\end{array}\right.\end{array}$ & $\begin{array}{c}\% \\
\text { vivants }\end{array}$ \\
\hline \multirow{2}{*}{$\begin{array}{l}\text { Groupe } \\
\text { expéri- } \\
\text { mental }\end{array}$} & 200 & 74 & $5,09^{* *}$ & $2,06 * *$ & 868 & $3.837^{* *}$ & 84,7 \\
\hline & 0 & 73 & 3,11 & 2,38 & 930 & 2.821 & 88,7 \\
\hline $\begin{array}{l}\text { Groupe } \\
\text { Témoin }\end{array}$ & 0 & 78 & 2,83 & 2,47 & 945 & 2.517 & 88,2 \\
\hline
\end{tabular}

** Significativement différent au niveau 1 p. 100. 
L'effet le plus remarquable est une augmentation importante du volume et du nombre total de spermatozoïdes. Par contre, la motilité et le taux de spermatozoïdes vivants sont abaissés et la concentration n'est pas significativement différente de celle des témoins. Aucune différence n'est observée entre les témoins et les animaux ayant reçu $200 \mathrm{mg}$ de pilocarpine trois ou quatre jours avant.

L'action obtenue sur la composition du sperme est particulièrement nette. L'augmentation de volume et du nombre total de spermatozoïdes est comparable à celle obtenue par BAKER, Van DEMARK et GRAVEs (I963).

Contrairement aux résultats de MinTschev (I963) qui note une disparition de tout comportement sexuel chez des chats traités à la piloca rpine, nous n'avons observé aucune modification dans ce domaine.

Il semble donc que l'innervation parasympathique joue un rôle essentiel aussi bien dans l'émission des constituants du plasma séminal lors de l'éjaculation, que dans celle des spermatozoïdes eux-mêmes.

Reçu pour publication en mars 1964 .

\section{SUMMARY}

ACTION OF PILOCARPINE ON THE COMPOSITION OF THE BULL SEMEN

Ten bulls were given 30 minutes before semen collection, a subcutaneous injection of either $200 \mathrm{mg}$ pilocarpine or physiological saline.

The sexual behaviour was normal but the characteristics of the semen were distinctly modified. Semen volume rises from 2,8 to $5,0 \mathrm{ml}$ and total sperm number increases from 2,5 to 3,8 billions $(\mathbf{P}<0,0 \mathbf{I})$.

The parasympathetic innervation of the genital tract seems very important in the mechanisms of ejaculation.

\section{RÉFÉRENCES BIBLIOGRAPHIQUES}

Baker R. D., Van Demark N. L., Graves C. N., 1963. Drug effects on behavior and ejaculates of Bulls (Abstr.). J. Anim. Sci., 22, г 136 .

BLom E., I950. A rapid staining method using Eosin-Nigrosin to distinguish between live and dead spermatozoa. Nord. Ve. Med., 2, 58-6I.

DzIUK P. J., I959. Semen characteristics and sexual behavior in the boar following administration of atropine. (Abstr.) J. Anim. Sci., 18, I 554-I 555 .

Dziuk P. J., NorTon II. W., I962. Influence of drugs affecting the autonomic system on seminal ejaculation. J. Reprod. Fertil., 4, 47-50.

Mintscilev P., I963. Die Beeinflussung des Geschlechtstriebes bei Katzen mit M.-cholinomimetika. Zuchthyg. Fortpil.-Siör. Besan. Hausitere, 7, $120-126$.

Signoret J. P., 1962. Action de l'atropine sur le comportement sexuel et la composition du sperme chez le Taureau. Ann. Biol. anim. Bioch. Biophys., 2, 163-166. 